\title{
An effective face recognition method using guided image filter and convolutional neural network
}

\author{
Yallamandaiah S., Purnachand N. \\ School of Electronics Engineering, VIT-AP University, Amaravati, Andhra Pradesh, India
}

\begin{abstract}
Article Info
Article history:

Received Jun 19, 2021

Revised Jul 23, 2021

Accepted Aug 10, 2021

\section{Keywords:}

Computer vision

Convolutional neural network

Face recognition

Guided image filter

ABSTRACT

In the area of computer vision, face recognition is a challenging task because of the pose, facial expression, and illumination variations. The performance of face recognition systems reduces in an unconstrained environment. In this work, a new face recognition approach is proposed using a guided image filter, and a convolutional neural network (CNN). The guided image filter is a smoothing operator and performs well near the edges. Initially, the ViolaJones algorithm is used to detect the face region and then smoothened by a guided image filter. Later the proposed CNN is used to extract the features and recognize the faces. The experiments were performed on face databases like ORL, JAFFE, and YALE and attained a recognition rate of $98.33 \%$, $99.53 \%$, and $98.65 \%$ respectively. The experimental results show that the suggested face recognition method attains good results than some of the state-of-the-art techniques.
\end{abstract}

This is an open access article under the CC BY-SA license.

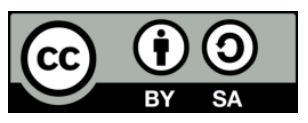

\section{Corresponding Author:}

Purnachand N.

School of Electronics Engineering

VIT-AP University

Amaravati, Andhra Pradesh 522237, India

Email: chanduinece@gmail.com

\section{INTRODUCTION}

The face is an important biological trait that differentiates an individual from others. Face recognition is a process of extracting features and identifying individuals, and it is an efficient approach among many biometrics because of its full automation and uniqueness [1]. Preprocessing, feature extraction, and classification are the three steps in the face recognition task. The extraction of features and the construction of the proper classifier play a major role in the face recognition process.

Face recognition has become popular because of its utility in different areas such as communication, file management, human-computer interaction, surveillance, security, and law enforcement. In particular, face recognition is widely used to identify missing babies, detect passport frauds, unlocking apps on mobile phones, and stop blacklisted persons in restaurants. The efficiency of the face recognition systems reduces because of variations in illuminations, poses, expressions of the person. In an uncontrolled environment, the face images are affected by different illuminations and noises. A guided image filter has a variety of applications such as noise reduction, haze removal, enhancement/smoothing [2]. Recently convolutional neural networks producing good results in the case of image classification. The tradeoff when moving from traditional approaches to deep learning approaches is training time i.e. initially it takes a long time for training the data to the $\mathrm{CNN}$, but the classification accuracy will be high compared to earlier methods. To deal with the various illuminations, poses, and expressions of the person, this paper proposes a new face recognition method using a guided image filter and convolutional neural network (CNN). 
The structure of the paper is planned as follows: Related work is discussed, in Section 2. In Section 3, the suggested face recognition method is explained. The experimental outcomes are demonstrated in Section 4. The conclusion of the paper is presented in Section 5.

\section{RELATED WORK}

Feature extraction and the construction of the classifier play a crucial role in the face recognition process. The principal component analysis (PCA) is a prominent method for feature extraction. Kirby and Sirovich used principal components to represent human faces [3]. Turk et al. utilized the plan of Ref. 3 for face detection and recognition [4]. PCA reduces the dimension and eliminates correlation, however, it is not appropriate for classification [5], [6]. Meedeniya and Ratnaveera proposed an enhanced face recognition method through the variation of PCA, in which the authors performed the economic size singular value decomposition to generate a unitary matrix [7].

Linear discriminant analysis (LDA) is an eminent dimensionality reduction method, but it fails when the count of training samples is less compared to the count of dimensions of the feature space [8]. To overcome this problem Belhumer et al. proposed the fisher faces method [9]. Yun and Ruan propounded enhanced fisher's linear discriminant (EFLD) method and it outperforms the earlier algorithms [10]. Zhou et al. suggested a face recognition method by combining a Non-Negative Matrix Factorization with a Radial Basis Function classifier [11]. Abusham et al. demonstrated an approach for face recognition by fusing Locally Linear Embedding and PCA [12]. Local binary patterns (LBP) [13] and local phase quantization (LPQ) [14], [15] have attained good face recognition implementation in constrained environments. The performance of these handcrafted features reduces considerably in an unconstrained environment. Zhou et al. introduced a face recognition method using PCA and LDA [16]. Dai et al. manifested a decorrelated 2Dfeed-forward neural network (2D-FNN) ensemble with random weights [17]. The feature-level fusion of local binary patterns and coefficient enhancement algorithms on contourlet-subbands made a robust expression invariant face recognition system [18]. Khan et al. proposed a system that can recognize faces under varying expressions and illumination using particle swarm optimization (PSO) [19]. Tai et al. proposed the orthogonal procrustes problem (OOP) as a framework to pose changes in face images [20]. Li et al. introduced a projective low-rank description method for face recognition [21]. Chen Y et al. addressed the problem of multi-pose classification using 2D-Gabor features and the Deep Belief Nets [22]. Yin et al. suggested multi-task learning for recognizing faces with the pose and expression estimation as the side tasks [23]. Ding et al. introduced an improved human activity recognition system based on a random forest classifier [24]. Liao et al. suggested a novel cluster multiple kernel learning algorithm for recognizing the oil painters [25]. Muqeet et al. presented a face recognition method by utilizing directional wavelet transform and local binary patterns [26].

In recent years CNN methods have grabbed substantial attentiveness in face recognition. The CNNs considerably enhances the model generation ability by establishing effective regularization strategies such as dropout [27]. The research group at Facebook developed a deep learning facial recognition system named DeepFace [28]. Wu et al. discovered the correlations among the sustainable development goals and communication technologies [29]. Various pattern recognition algorithms for human activity recognition were reviewed and discussed in [30]. Lin et al. propounded a new robust dictionary learning approach for face recognition [31]. Georgel et al. used deep stacked de-noising and sparse auto-encoders (DSDSA) for face recognition [32]. In this work, a new face recognition technique is proposed using a guided image filter and a convolutional neural network.

\section{PROPOSED WORK}

This work proposes a new approach for face recognition using a guided image filter and a CNN. The proposed method consists of the following steps: face detection, image resizing, applying guided image filter on the resized image, extracting features, and recognizing faces with the help of the proposed CNN. Initially, the face region is extracted using the Viola-Jones algorithm and resized to $128 \mathrm{x} 128$. The entire methodology is depicted in Figure 1.

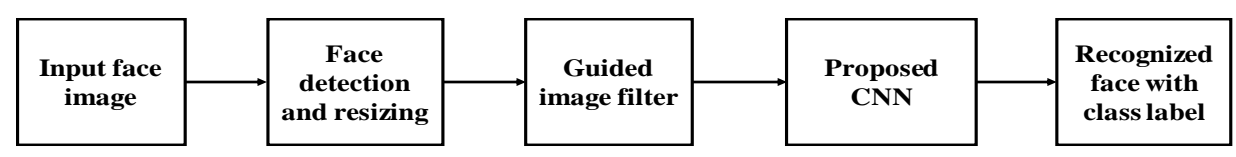

Figure 1. Block diagram of the proposed face recognition method 


\subsection{Guided image filter}

To obtain information in images, the majority of applications in pattern recognition uses image filtering. The mean, Laplacian, Sobel, and Gaussian filters have been extensively utilized in image feature extraction, sharpening/blurring, restoration and edge detection. The bilateral filter is the most intuitive and simplest one among weighted average filters [33]. Even though the bilateral filter is successful in many circumstances, gradient reversal artifacts diminish its performance [34]-[36]. This problem is overcome by the guided filter. The guided filter is an explicit image filter obtained from a linear model and determines the filtering output based upon the content of the guidance image [37].

Let the $I$ is a guidance image, and $p$ is a filtering input image, the general linear translation-invariant filtering outcome at a pixel $i$ is given as,

$$
q_{i}=\sum_{j} W_{i j}(I) p_{j}
$$

where $i$, and $j$ are pixel indices, $W_{i j}$ is the kernel.

The guided filter is a linear model between guidance $I$ and the filtering output $q$ and is given by:

$$
q_{i}=a_{k} I_{i}+b_{k}, \forall i \in w_{k}
$$

where $\left(a_{k}, b_{k}\right)$ are linear coefficients in $w_{k}$.

To determine the coefficients $\left(a_{k}, b_{k}\right)$, the output $q$ is modeled as the input $p$ subtracting few undesirable components $n$ :

$$
q_{i}=p_{i}-n_{i}
$$

Minimize the following cost function in the window $w_{k}$ to find the solution for the (2)

$$
E\left(a_{k}, b_{k}\right)=\sum_{i \in w_{k}}\left(\left(a_{k} I_{i}+b_{k}-p_{i}\right)^{2}+\varepsilon a_{k}^{2}\right)
$$

where $\varepsilon$ is a regularization parameter. In (4) is the linear regressive model and its solution is given by,

$$
\begin{aligned}
& a_{k}=\frac{\frac{1}{|w|} \sum_{i \in w_{k}} I_{i} p_{i}-\mu_{k} \bar{p}_{k}}{\sigma_{k}^{2}+\varepsilon} \\
& b_{k}=\bar{p}_{k}-a_{k} \mu_{k}
\end{aligned}
$$

Here $\mu_{k}$ and $\sigma_{k}^{2}$ are the mean and variance of $I$ in $w_{k},\left|w_{k}\right|$ is the total number of pixels in $w_{k}$ and $\bar{p}_{k}=\frac{1}{|w|} \sum_{i \in w_{k}} p_{i}$ is the mean of $p$ in $w_{k}$. After obtaining $\left(a_{k}, b_{k}\right)$, we can find the filtering output $q_{i}$ by (2). The input face image and the filtered output from the guided image filter are shown in Figure 2.

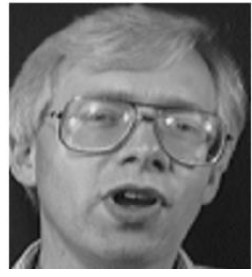

(a)

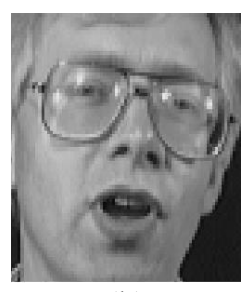

(b)

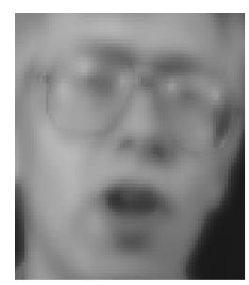

(c)

Figure 2. (a) Input face image (b) Face detection (c) Guided image filter output

\subsection{Proposed convolutional neural network}

In recent years convolutional neural networks have remarkably boosted the state-of-the-art performance for various visual tasks. For example, image retrieval, semantic segmentation, multitask learning, image classification, and person re-identification. Convolutional neural networks integrate both feature extraction and classification. The feature extraction is done by the convolutional layer and pooling layer. The fully connected layer is used for classification purposes. 
The architecture of the proposed CNN is depicted in Figure 3. The proposed CNN consists of four convolutional layers with 8, 16, 32, and 64 filters. ReLU nonlinear activation function is utilized in each convolutional layer. In each convolutional layer, the stride is set to one. The input to the proposed $\mathrm{CNN}$ is a $128 \times 128 \times 1$ image. The first convolutional layer consists of $5 \times 5$ kernels with eight filters. Hence the outcome of the conv1 is eight feature maps with size $124 \times 124$. Max pooling layers follow each convolutional layer with a $2 \times 2$ window and stride two. Maxpooling 1 produces feature maps with a size of $62 \times 62$. In each maxpooling layer, the stride is set to two. The dimensions of the feature maps generated by conv2, conv3, and

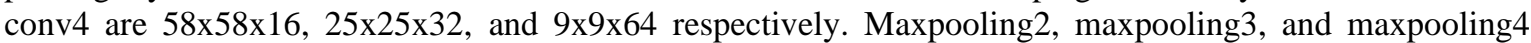
layers produce an output with dimensions $29 \times 29 \times 16,13 \times 13 \times 32$, and $4 \times 4 \times 64$ respectively. The final maxpooling layer is followed by two fully connected layers with 1024 and 512 units. Finally, the softmax function is used for classification purposes. Stochastic Gradient Descent is utilized as an optimizer for training the data to the proposed $\mathrm{CNN}$, with a base learning rate of 0.001 . we used a batch size of four while training the network.

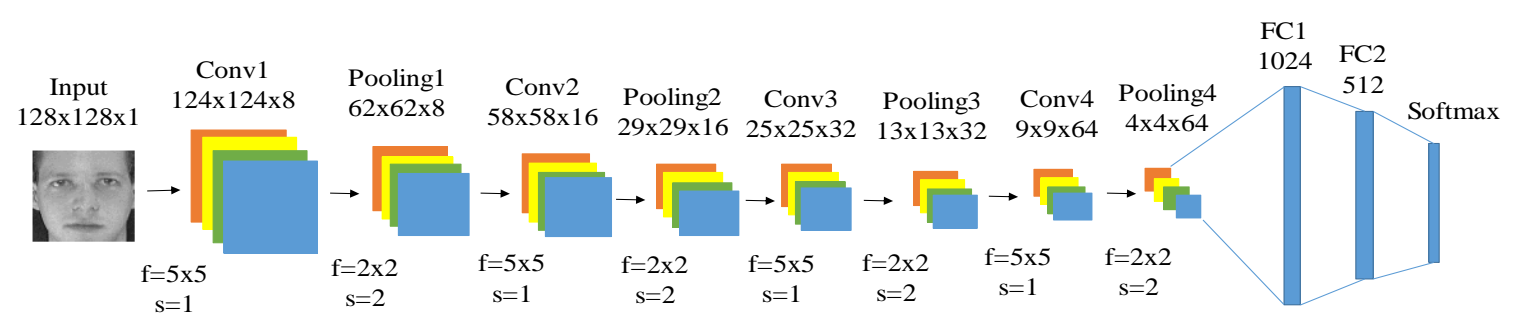

Figure 3. Architecture of the proposed CNN

\subsection{The novelty of the proposed method}

The novelty of the proposed methodology is removing the noise present in the face images before applying them to the proposed CNN and designing the CNN with a limited number of layers. Removing the noises existing in the face images improves the face recognition accuracy and designing the CNN with a fewer number of layers decreases the model complexity. Appending extra layers help to extract the more detailed features, but we can add layers up to a certain limit. After that, the model overfits the data which leads to errors like false positives. In addition to this, if we add more layers the number of weights in the network increases and leads to increase in the model complexity. To reduce this complexity, we designed the CNN with the optimum number of layers.

\section{EXPERIMENTAL RESULTS AND DISCUSSIONS}

We show the efficiency of our face recognition system across different poses, expressions, and illuminations using the ORL [38], JAFFE [39], and YALE [40] face datasets. We tested our method for a different radius $(r)$ of the square window and regularization parameters $(\varepsilon)$ of a guided image filter. We choose $70 \%$ of images in each class for training and the rest of the images were utilized for testing.

The ORL face database comprises 400 face images collected from 40 different persons with ten distinct images for each person. These images manifest variations in the pose, illumination, and facial expressions like smiling or not smiling, eyes closed or opened. The JAFFE face database is a collection of 213 grayscale images of ten Japanese female models. The database comprises facial expressions like the surprise, happiness, sadness, anger, fear, neutral, and disgust of each subject. The YALE face database includes 165 grayscale images of 15 subjects with 11 images per subject. Each subject contains images with the following configurations: center-light, happy, with glasses, sleepy, left-light, normal, sad, with no glasses, wink, rightlight, and surprised. The images that belong to a single subject of these databases are shown in Figure 4.

Figure 5 shows the output images of the guided image filter for ORL, JAFFE, and YALE databases with different sets of parameters. For comparison purposes, we extracted the features from the second fully connected layer of the proposed $\mathrm{CNN}$ and classified them using a decision tree and random forest classifier. The efficiency of the classifier is evaluated using recognition rate (RR), area under curve (AUC), true positive rate (TPR), and false positive rate (FPR). The RR of the proposed method for the different radius (r) of the square window and regularization parameter $(\varepsilon)$ is given in Table 1 . The suggested method gives the best RR of $98.33 \%, 99.53 \%$, and $98.65 \%$ on ORL, JAFFE, and YALE databases respectively for $r=2$ and $\varepsilon=0.4^{2}$. Compared to the random forest and decision tree classifiers softmax has given the best RR. 
Table 2 gives the TPR for all the chosen face databases. The softmax classifier has given a maximum TPR of $98.57 \%$ whereas the decision tree and random forest classifiers have produced TPR of $99.61 \%$ and $98.65 \%$ on ORL, JAFFE, and YALE databases respectively for $r=2$ and $\varepsilon=0.4^{2}$. From Table 2, it is noticed that the softmax classifier has given high TPR compared to the decision tree and random forest. The FPR for the proposed method with different classifiers is given in Table 3. Low FPR is attained with a softmax classifier compared to the decision tree and random forest classifiers. The proposed method with softmax classifier has given an FPR of 0.02, 0.01, and 0.02 on ORL, JAFFE, and YALE databases for $r=2$ and $\varepsilon=$ $0.4^{2}$. The AUC is determined and given in Table 4. The maximum value of the AUC with softmax classifier on ORL, JAFFE, and YALE databases is $99.52 \%, 99.78 \%$, and $99.63 \%$. From the values of Table 4, it is observed that the proposed method with the softmax classifier has more AUC than the other classifiers.

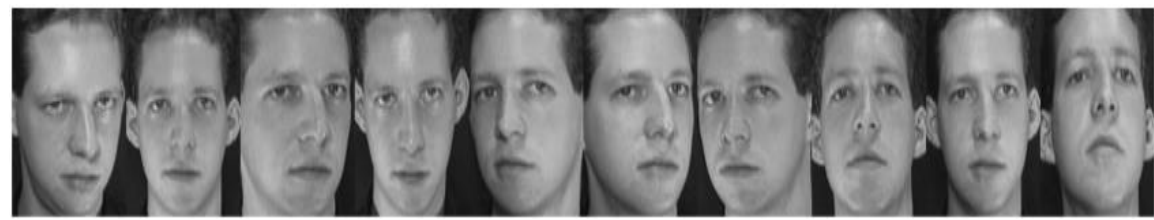

(a)
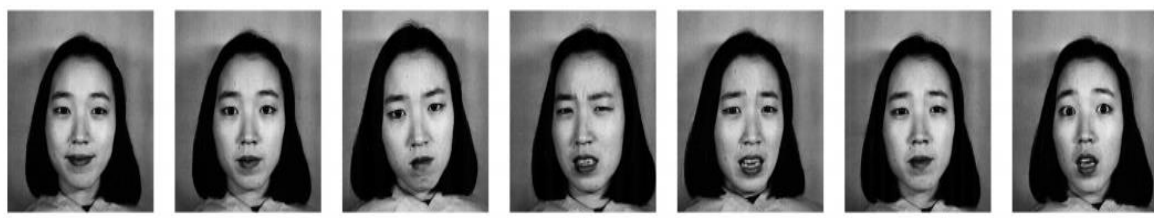

(b)

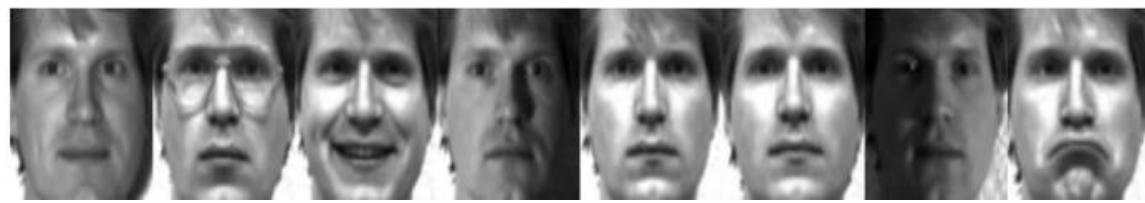

(c)

Figure 4. Images belong to a single subject of (a)ORL, (b)JAFFE and (c)YALE databases
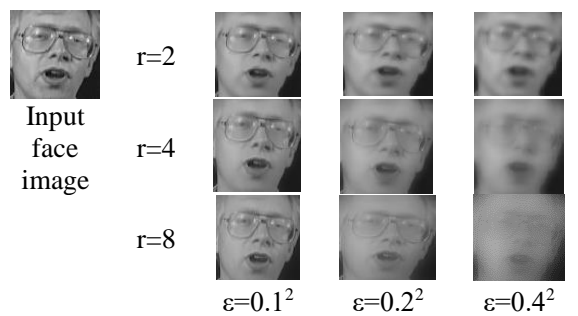

(a)
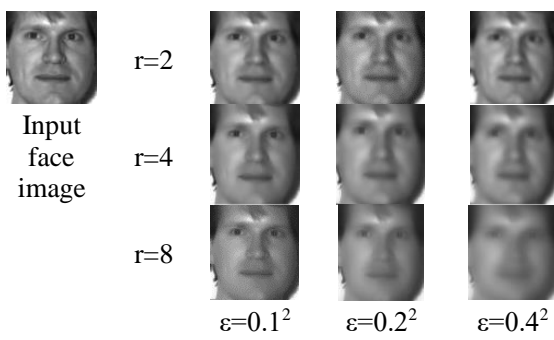

(c)
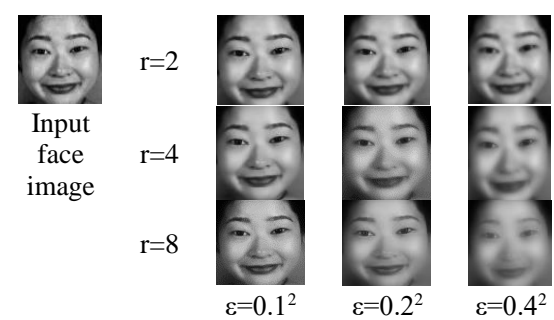

(b)

Figure 5. The output images of a guided image filter for the gray-scale input from (a) ORL (b) JAFFE and (c) YALE database 
Table 1. Recognition rate using decision tree, random forest, and softmax classifier

\begin{tabular}{|c|c|c|c|c|c|c|c|c|c|c|}
\hline $\begin{array}{l}\text { Radius } \\
\text { (r) }\end{array}$ & $\begin{array}{l}\text { Regularization } \\
\text { parameter }(\varepsilon)\end{array}$ & \multicolumn{3}{|c|}{ ORL } & \multicolumn{3}{|c|}{ JAFFE } & \multicolumn{3}{|c|}{ YALE } \\
\hline \multirow{2}{*}{2} & $0.1^{2}$ & 95.25 & 95.87 & 96.67 & 94.28 & 95.52 & 98.33 & 95.17 & 95.28 & 97.78 \\
\hline & $0.4^{2}$ & 95.43 & 96.58 & 98.33 & 95.82 & 96.74 & 99.53 & 95.63 & 96.62 & 98.65 \\
\hline \multirow[b]{2}{*}{4} & $0.1^{2}$ & 86.23 & 88.19 & 89.17 & 92.59 & 93.33 & 96.67 & 91.24 & 93.48 & 97.89 \\
\hline & $0.2^{2}$ & 87.62 & 89.45 & 91.67 & 91.35 & 94.63 & 96.67 & 92.87 & 94.24 & 97.78 \\
\hline \multirow[t]{2}{*}{8} & $0.2^{2}$ & 86.67 & 87.86 & 90.83 & 89.82 & 92.12 & 93.33 & 91.73 & 94.66 & 97.78 \\
\hline & $0.4^{2}$ & 85.41 & 88.38 & 90.00 & 90.91 & 91.53 & 93.33 & 87.24 & 89.15 & 93.33 \\
\hline
\end{tabular}

Table 2. True positive rate using decision tree, random forest, and softmax classifier

\begin{tabular}{|c|c|c|c|c|c|c|c|c|c|c|}
\hline \multirow[b]{2}{*}{$\begin{array}{l}\text { Radius } \\
\text { (r) }\end{array}$} & \multirow[b]{2}{*}{$\begin{array}{c}\text { Regularization } \\
\text { parameter }(\varepsilon)\end{array}$} & \multicolumn{3}{|c|}{ ORL } & \multicolumn{3}{|c|}{ JAFFE } & \multicolumn{3}{|c|}{ YALE } \\
\hline & & $\begin{array}{c}\text { Decision } \\
\text { Tree }\end{array}$ & $\begin{array}{c}\text { Random } \\
\text { Forest }\end{array}$ & Softmax & $\begin{array}{c}\text { Decision } \\
\text { Tree } \\
\end{array}$ & $\begin{array}{c}\text { Random } \\
\text { Forest }\end{array}$ & Softmax & $\begin{array}{c}\text { Decision } \\
\text { Tree } \\
\end{array}$ & $\begin{array}{c}\text { Random } \\
\text { Forest }\end{array}$ & Softmax \\
\hline \multirow{2}{*}{2} & $0.1^{2}$ & 95.25 & 95.87 & 96.67 & 94.28 & 95.52 & 98.33 & 95.17 & 95.28 & 97.78 \\
\hline & $0.4^{2}$ & 95.43 & 96.88 & 98.57 & 95.82 & 96.94 & 99.61 & 95.63 & 96.93 & 98.65 \\
\hline \multirow[b]{2}{*}{4} & $0.1^{2}$ & 86.23 & 88.19 & 90.24 & 93.25 & 93.33 & 96.67 & 91.24 & 93.48 & 98.21 \\
\hline & $0.2^{2}$ & 87.62 & 89.45 & 91.67 & 91.35 & 94.63 & 96.67 & 93.52 & 95.26 & 97.78 \\
\hline \multirow[t]{2}{*}{8} & $0.2^{2}$ & 86.92 & 87.86 & 90.83 & 89.82 & 92.12 & 94.36 & 92.13 & 94.89 & 97.78 \\
\hline & $0.4^{2}$ & 85.41 & 88.38 & 91.62 & 90.91 & 91.53 & 94.36 & 87.24 & 89.15 & 94.73 \\
\hline
\end{tabular}

Table 3. False positive rate using decision tree, random forest, and softmax classifier

\begin{tabular}{|c|c|c|c|c|c|c|c|c|c|c|}
\hline $\begin{array}{l}\text { Radius } \\
\text { (r) }\end{array}$ & $\begin{array}{l}\text { Regularization } \\
\text { parameter }(\varepsilon)\end{array}$ & \multicolumn{3}{|c|}{ ORL } & \multicolumn{3}{|c|}{ JAFFE } & \multicolumn{3}{|c|}{ YALE } \\
\hline \multirow{3}{*}{2} & $0.1^{2}$ & 0.06 & 0.04 & 0.03 & 0.06 & 0.05 & 0.02 & 0.07 & 0.04 & 0.03 \\
\hline & $0.4^{2}$ & 0.06 & 0.03 & 0.02 & 0.06 & 0.04 & 0.01 & 0.07 & 0.04 & 0.02 \\
\hline & $0.1^{2}$ & 0.09 & 0.08 & 0.07 & 0.09 & 0.08 & 0.04 & 0.08 & 0.05 & 0.03 \\
\hline 4 & $0.2^{2}$ & 0.05 & 0.04 & 0.06 & 0.09 & 0.07 & 0.04 & 0.07 & 0.06 & 0.03 \\
\hline \multirow[t]{2}{*}{8} & $0.2^{2}$ & 0.09 & 0.09 & 0.07 & 0.08 & 0.07 & 0.05 & 0.07 & 0.06 & 0.03 \\
\hline & $0.4^{2}$ & 0.09 & 0.08 & 0.06 & 0.07 & 0.04 & 0.05 & 0.08 & 0.07 & 0.05 \\
\hline
\end{tabular}

Table 4. Area under curve using decision tree, random forest, and softmax classifier

\begin{tabular}{|c|c|c|c|c|c|c|c|c|c|c|}
\hline \multirow[b]{2}{*}{$\begin{array}{l}\text { Radius } \\
\text { (r) }\end{array}$} & \multirow[b]{2}{*}{$\begin{array}{l}\text { Regularization } \\
\text { parameter }(\varepsilon)\end{array}$} & \multicolumn{3}{|c|}{ ORL } & \multicolumn{3}{|c|}{ JAFFE } & \multicolumn{3}{|c|}{ YALE } \\
\hline & & $\begin{array}{c}\text { Decision } \\
\text { Tree }\end{array}$ & $\begin{array}{l}\text { Random } \\
\text { Forest }\end{array}$ & softmax & $\begin{array}{c}\text { Decision } \\
\text { Tree }\end{array}$ & $\begin{array}{c}\text { Random } \\
\text { Forest }\end{array}$ & softmax & $\begin{array}{c}\text { Decision } \\
\text { Tree }\end{array}$ & $\begin{array}{c}\text { Random } \\
\text { Forest }\end{array}$ & softmax \\
\hline \multirow{3}{*}{2} & $0.1^{2}$ & 96.53 & 97.84 & 98.73 & 95.94 & 96.45 & 99.53 & 96.67 & 97.59 & 99.24 \\
\hline & $0.4^{2}$ & 96.82 & 97.23 & 99.52 & 97.95 & 98.92 & 99.78 & 96.92 & 97.63 & 99.63 \\
\hline & $0.1^{2}$ & 87.42 & 88.44 & 93.72 & 93.48 & 95.47 & 98.75 & 93.67 & 96.98 & 98.76 \\
\hline 4 & $0.2^{2}$ & 88.85 & 90.28 & 95.38 & 94.78 & 95.58 & 98.75 & 94.27 & 98.83 & 99.24 \\
\hline \multirow[t]{2}{*}{8} & $0.2^{2}$ & 87.76 & 88.43 & 94.68 & 92.28 & 95.76 & 98.47 & 93.66 & 97.44 & 99.21 \\
\hline & $0.4^{2}$ & 86.45 & 89.92 & 93.72 & 94.19 & 96.29 & 98.47 & 91.34 & 95.52 & 98.78 \\
\hline
\end{tabular}

The ROC curves for the proposed technique with softmax classifiers are depicted in Figure 6 . The performance comparison of the suggested method with some of the existing methods is encapsulated in Table 5. From the values of Table 5, it is noticed that the proposed face recognition approach gives a good recognition rate than some of the earlier approaches.

Image data augmentation enlarges the size of the training dataset by producing the modified versions of the images in the dataset. Data augmentation can improve the performance of the proposed face recognition system. We carried out horizontal and vertical flipping, rotation by 45 degrees, and zooming for generating additional images. The recognition rate of the proposed method with and without the data augmentation is given in Table 6. 


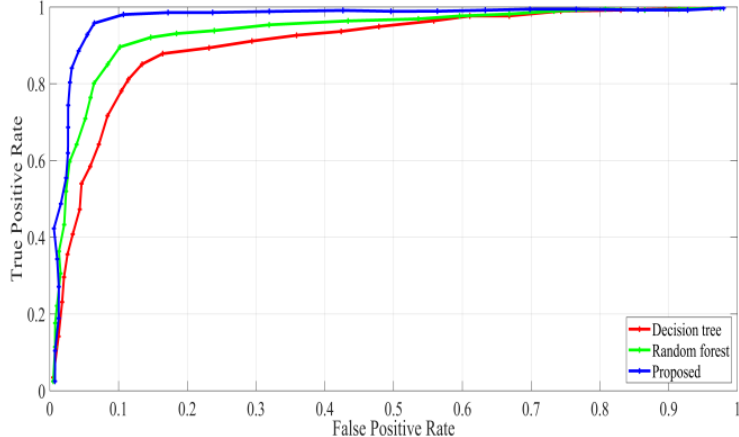

(a)

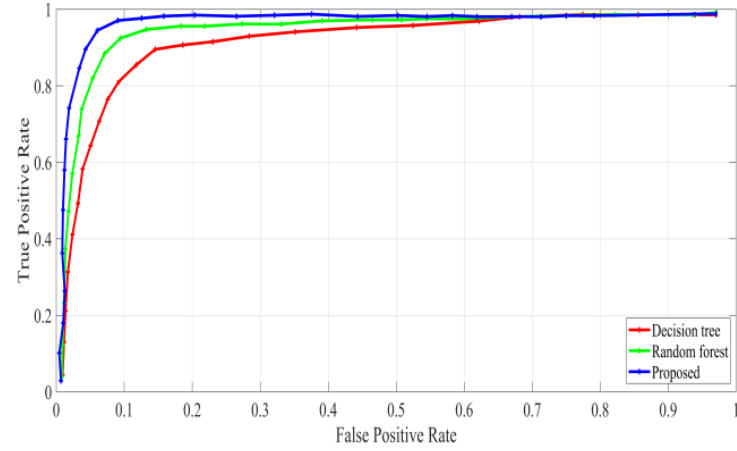

(b)

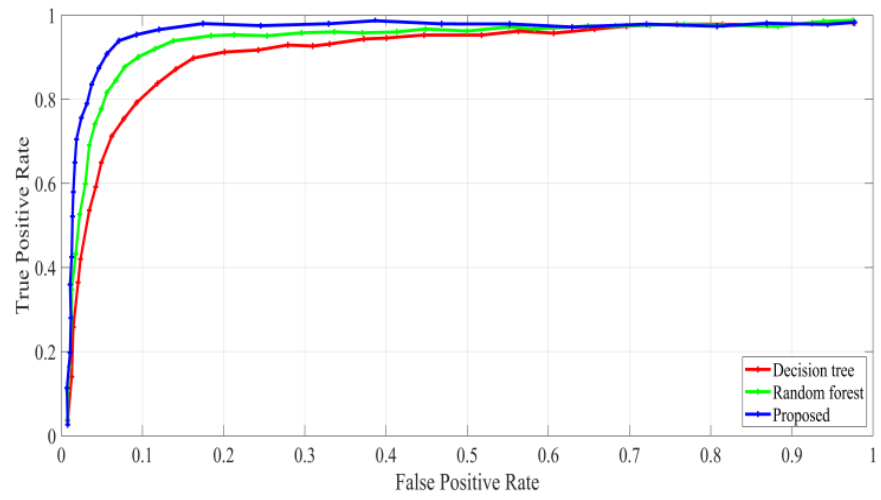

(c)

Figure 6. ROC curves for (a) ORL (b) JAFFE and (c) YALE databases for $r=2$ and $\varepsilon=0.4^{2}$

Table 5. Comparison of recognition rate (\%) with some of the existing techniques

\begin{tabular}{cccc}
\hline Method & ORL & $\begin{array}{l}\text { Database } \\
\text { JAFFE }\end{array}$ & YALE \\
\hline CLDA [5] & 94.06 & & \\
PCA [8] & 89.50 & & \\
FLD [8] & 91.00 & & \\
PCA image reconstruction + LDA [14] & 97.20 & & \\
GFDBN [20] & 94.98 & & \\
DIWTLBP [22] & 97.00 & & \\
DSDSA [24] & 98.00 & & \\
Proposed & $\mathbf{9 8 . 3 3}$ & & \\
FLLEPCA [10] & & 94.98 & \\
Single 2D NNRW [15] & & 97.00 & \\
PSO [17] & & 98.80 & \\
Proposed & & $\mathbf{9 9 . 5 3}$ & \\
OPR [18] & & & 94.15 \\
PLR [19] & & & 97.23 \\
RDCDL [23] & & & 98.16 \\
DSDSA [24] & & & $\mathbf{9 8 . 6 5}$ \\
Proposed & &
\end{tabular}

Table 6. Recognition rate of the proposed method with and without data augmentation

\begin{tabular}{cccc} 
& \multicolumn{3}{c}{ Database } \\
& ORL & JAFFE & YALE \\
\hline Without Data Augmentation & $98.33 \%$ & $99.53 \%$ & $98.65 \%$ \\
With Data Augmentation & $99.17 \%$ & $99.74 \%$ & $99.32 \%$ \\
\hline
\end{tabular}

\section{CONCLUSION}

In this work, a face recognition approach using a guided image filter and a convolutional neural network was proposed. First, the face region of the test image is extracted using the Viola-Jones algorithm and then resized. The resized image was passed through a guided image filter. A guided image filter 
smoothens the face image, then the proposed CNN was utilized to extract the features and classify the input face image. Here, the softmax classifier, which gives good results than the decision tree and random forest, was used in CNN's classifier section. The capability of the proposed approach was compared with some of the earlier methods. From the comparative results, it is found that the suggested technique produces better results than some of the existing methods. Based on the experimental results, it is concluded that our proposed method can be used for face recognition.

\section{REFERENCES}

[1] Yang, Ming-Hsuan, David J. Kriegman, and Narendra Ahuja, "Detecting faces in images: A survey," IEEE Transactions on pattern analysis and machine intelligence, vol. 24, no. 1, pp. 34-58, 2002, doi: 10.1109/34.982883.

[2] He, Kaiming, Jian Sun, and Xiaoou Tang, "Guided image filtering," European conference on computer vision. Springer, Berlin, Heidelberg, 2010, doi: 10.1109/TPAMI.2012.213.

[3] Kirby, Michael, and Lawrence Sirovich, "Application of the Karhunen-Loeve procedure for the characterization of human faces," IEEE Transactions on Pattern analysis and Machine intelligence, vol. 12, no. 1, pp. 103-108, 1990.

[4] Turk Matthew, and Alex Pentland, "Eigenfaces for recognition," Journal of cognitive neuroscience, vol. 3, no. 1 pp. 71-86, 1991, doi: 10.1162/jocn.1991.3.1.71.

[5] Zhao, Wenyi, A Krishnaswamy, Rama Chellappa, Daniel L. Swets, and John Weng, "Discriminant analysis of principal components for face recognition," Face Recognition. Springer, Berlin, Heidelberg, pp. 73-85, 1998, doi: 10.1109/AFGR.1998.670971.

[6] Lu, Gui-Fu, Jian Zou, and Yong Wang, "Incremental complete LDA for face recognition," Pattern Recognition, vol. 45, no. 7, pp. 2510-2521, 2012, doi: 10.1016/j.patcog.2012.01.018.

[7] D. A. Meedeniya, and D. A. A. C. Ratnaweera, "Enhanced face recognition through variation of principle component analysis (PCA)," 2007 International Conference on Industrial and Information Systems, IEEE, 2007, doi: 10.1109/ICIINFS.2007.4579200.

[8] Bansal, Abhishek, Kapil Mehta, and Sahil Arora, "Face recognition using PCA and LDA algorithm," 2012 second international conference on Advanced Computing \& Communication Technologies. IEEE, 2012, doi: 10.1109/ACCT.2012.52.

[9] Belhumeur, Peter N., João P. Hespanha, and David J. Kriegman, "Eigenfaces vs. fisherfaces: Recognition using class specific linear projection," IEEE Transactions on pattern analysis and machine intelligence, vol. 19, no. 7, pp. 711-720, 1997, doi: 10.1007/BFb0015522.

[10] An, GaoYun, and QiuQi Ruan, "Novel mathematical model for enhanced fisher's linear discriminant and its application to face recognition," 18th International Conference on Pattern Recognition (ICPR'06), IEEE, vol. 2, 2006, pp. 524-527, doi: 10.1109/ICPR.2006.873.

[11] Zhou, Wei, Xiaorong Pu, and Ziming Zheng, "Parts-based holistic face recognition with RBF neural networks," International Symposium on Neural Networks, Springer, Berlin, Heidelberg, 2006, doi: $10.1007 / 1176002317$.

[12] Abusham, Eimad Eldin, David Ngo, and Andrew Teoh, "Fusion of locally linear embedding and principal component analysis for face recognition (FLLEPCA)," International Conference on Pattern Recognition and Image Analysis. Springer, Berlin, Heidelberg, 2005, doi: 10.1007/11552499_37.

[13] Ahonen, Timo, Abdenour Hadid, and Matti Pietikäinen, "Face recognition with local binary patterns," European conference on computer vision. Springer, Berlin, Heidelberg, 2004.

[14] Ahonen Timo, Esa Rahtu, Ville Ojansivu, and Janne Heikkila, "Recognition of blurred faces using local phase quantization," 2008 19th international conference on pattern recognition. IEEE, 2008, doi: 10.1109/ICPR.2008.4761847.

[15] Chi Ho Chan, M A Tahir, Josef Kittler, and M Pietikainen, "Multiscale local phase quantization for robust component-based face recognition using kernel fusion of multiple descriptors," IEEE Transactions on Pattern Analysis and Machine Intelligence, vol. 35, no. 5, pp. 1164-1177, 2012, doi: 10.1109/TPAMI.2012.199.

[16] Zhou Changjun, L Wang, Q Zhang, and X Wei, "Face recognition based on PCA image reconstruction and LDA," Optik, vol. 124, no. 22, pp. 5599-5603, 2013, doi: 10.1016/j.ijleo.2013.04.108.

[17] Dai, Kankan, Jianwei Zhao, and Feilong Cao, "A novel decorrelated neural network ensemble algorithm for face recognition," Knowledge-Based Systems, vol. 89, pp. 541-552, 2015, doi: 10.1016/j.knosys.2015.09.002.

[18] Patil, Hemprasad Y., Ashwin G. Kothari, and Kishor M. Bhurchandi, "Expression invariant face recognition using local binary patterns and contourlet transform," Optik, vol. 127, no. 5, pp. 2670-2678, 2016, doi: 10.1016/j.ijleo.2015.11.187.

[19] Khan Sajid Ali, M Ishtiaq, M Nazir, and M Shahneen, "Face recognition under varying expressions and illumination using particle swarm optimization," Journal of computational science, vol. 28, pp. 94-100, 2018, doi: 10.1016/j.jocs.2018.08.005.

[20] Tai Ying, J Yang, Y Zhang, L Luo, J Qian, and Y Chen, "Face recognition with pose variations and misalignment via orthogonal procrustes regression," IEEE Transactions on Image Processing, vol. 25, no. 6, pp. 2673-2683, 2016, doi: 10.1109/TIP.2016.2551362.

[21] Li Jun, Yu Kong, H Zhao, J Yang, and Yun Fu, "Learning fast low-rank projection for image classification," IEEE Transactions on Image Processing, vol. 25, no. 10, pp. 4803-4814, 2016, doi: 10.1109/TIP.2016.2598654.

[22] Chen Yong, T Huang, H Liu, and Di Zhan, "Multi-pose face ensemble classification aided by Gabor features and deep belief nets," Optik, vol. 127, no. 2, pp. 946-954, 2016, doi: 10.1016/j.ijleo.2015.10.179. 
[23] Yin, Xi, and Xiaoming Liu, "Multi-task convolutional neural network for pose-invariant face recognition," IEEE Transactions on Image Processing, vol. 27, no. 2, pp. 964-975, 2017, doi: 10.1109/TIP.2017.2765830.

[24] Genming Ding, Jun Tian, Jinsong Wu, Qian Zhao, and Lili Xie, "Energy efficient human activity recognition using wearable sensors," 2018 IEEE Wireless Communications and Networking Conference Workshops (WCNCW). IEEE, 2018, doi: 10.1109/WCNCW.2018.8368980.

[25] Zhifang Liao, Le Gao, Tian Zhou, Xiaoping Fan, Yan Zhang, and Jinsong Wu, "An oil painters recognition method based on cluster multiple kernel learning algorithm," IEEE Access, vol. 7, pp. 26842-26854, 2019, doi: 10.1109/ACCESS.2019.2899389.

[26] Muqeet, Mohd Abdul, and Raghunath S. Holambe, "Local binary patterns based on directional wavelet transform for expression and pose-invariant face recognition," Applied Computing and Informatics, vol. 15, no. 2, pp. 163171, 2019, doi: 10.1016/j.aci.2017.11.002.

[27] Geoffrey E. Hinton, N Srivastava, A Krizhevsky, I Sutskever, and R R. Salakhutdinov, "Improving neural networks by preventing co-adaptation of feature detectors," arXiv preprint arXiv:1207.0580, 2012.

[28] Taigman Yaniv, M Yang, M A Ranzato, and Lior Wolf, "Deepface: Closing the gap to human-level performance in face verification," Proceedings of the IEEE conference on computer vision and pattern recognition, 2014, doi: 10.1109/CVPR.2014.220.

[29] Jinsong Wu, Song Guo, Huawei Huang, William Liu, and Yong Xiang, "Information and communications technologies for sustainable development goals: state-of-the-art, needs and perspectives," IEEE Communications Surveys \& Tutorials, vol. 20, no. 3, pp. 2389-2406, 2018, doi: 10.1109/COMST.2018.2812301.

[30] Rachad Atat, Lingjia Liu, Jinsong Wu, Guangyu Li, Chunxuan Ye, and Yang Yi, "Big data meet cyber-physical systems: A panoramic survey," IEEE Access, vol. 6, pp. 73603-73636, 2018.

[31] Guojun Lin, M Yang, J Yang, L Shen, and W Xie, "Robust, discriminative and comprehensive dictionary learning for face recognition," Pattern Recognition, vol. 81, pp. 341-356, 2018, doi: 10.1016/j.patcog.2018.03.021.

[32] Görgel, Pelin, and Ahmet Simsek, "Face recognition via deep stacked denoising sparse autoencoders (DSDSA)," Applied Mathematics and Computation, vol. 355, pp. 325-342, 2019, doi: 10.1016/j.amc.2019.02.071.

[33] Tomasi, Carlo, and Roberto Manduchi, "Bilateral filtering for gray and color images," Sixth international conference on computer vision (IEEE Cat. No. 98CH36271). IEEE, 1998, doi: 10.1109/ICCV.1998.710815.

[34] Durand, Frédo, and Julie Dorsey, "Fast bilateral filtering for the display of high-dynamic-range images," Proceedings of the 29th annual conference on Computer graphics and interactive techniques, 2002, doi: $10.1145 / 566654.566574$.

[35] Bae, Soonmin, Sylvain Paris, and Frédo Durand, "Two-scale tone management for photographic look," ACM Transactions on Graphics (TOG), vol. 25, no. 3, pp. 637-645, 2006, doi: 10.1145/1141911.1141935.

[36] Zeev Farbman, Raanan Fattal, Dani Lischinski, and Richard Szeliski, "Edge-preserving decompositions for multiscale tone and detail manipulation," ACM Transactions on Graphics (TOG), vol. 27, no. 3, pp. 1-10, 2008, doi: $10.1145 / 1360612.1360666$

[37] He, Kaiming, Jian Sun, and Xiaoou Tang, "Guided image filtering," IEEE transactions on pattern analysis and machine intelligence, vol. 35, no. 6, pp. 1397-1409, 2012, doi: 10.1109/TPAMI.2012.213.

[38] O. Olivetti, "Oracle research laboratory face database of faces,"

[39] M. J. Lyons, S. Akamatsu, M. Kamachi, J. Gyoba, and J. Budynek, "The japanese female facial expression (jaffe) database," Proceedings of third international conference on automatic face and gesture recognition, 1998, pp. 14-16.

[40] P. N. Belhumeur., J. P. Hespanha., and D. J. Kriegman, "Eigenfaces vs.fisherfaces: Recognition using class specific linear projection," IEEE Transactions on Pattern Analysis \& Machine Intelligence, vol. 7, pp. 711-720, 1997.

\section{BIOGRAPHIES OF AUTHORS}
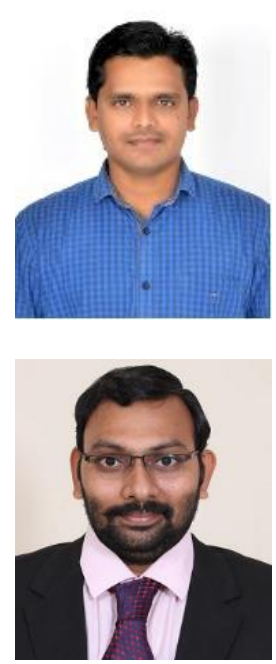

Yallamandaiah S received his B. Tech degree from JNTUH, Hyderabad, and M. Tech degree from JNTUK, Kakinada. Currently, he is a Research Scholar at the SENSE, VIT-AP University, India. His research fields include Image Processing and Machine Learning.

Purnachand $\mathbf{N}$ received his M.Tech degree from VIT University, Vellore, India, and $\mathrm{PhD}$ degree from University of Aveiro, Aveiro Portugal. Currently, he is working as Associate Professor at VIT-AP University, India. His fields of research contain Image Processing, Video Processing, and Pattern Recognition. 